\title{
DIPOLE NEAR FIELD ANALYSIS - A CLOSED FORM CALCULATION IN CARTESIAN COORDINATES
}

\author{
René Hart'anský — Jozef Slížik — Lukáš Maršálka *
}

\begin{abstract}
This article deals with analytical calculation of the near-field of an electric dipole in Cartesian coordinate system in a closed form. Results of the analytical calculation are verified by numerical method.

K e y w or ds: half-wave dipole, near field, analytical methods, numerical simulation
\end{abstract}

\section{INTRODUCTION}

In many areas of an electrical engineering like measurement of the electromagnetic fields, antenna theory, etc one needs to quantify mutual effect between conductive elements which are in the electromagnetic field. We rely upon the EMF method, [1] as a classical method to compute self and mutual impedances. The induced opencircuit voltage (output) in conductive element 2 , due to the radiation from conductive element 1 carrying current (input). The radiated field of conductive element 1 is usually calculated in cylindrical coordinates and the current distribution in conductive element 2 is estimated using antenna theory. Conductive elements are often arranged perpendicularly to a plane. If we want calculate the mutual impedance of such elements placed randomly in the space, more suitable may be the problem formulation and the near-field evaluation in Cartesian coordinates, particularly if we focus on the radial component. Besides this, the computation can be done without any approximation in a closed form - as will be shown here.

\section{CALCULATION OF NEAR FIELD OF THE ELECTRIC DIPOLE}

The calculation of the electric dipole near-field based on the geometry given in Fig. 1 , where point $M[x, y, z]$ is the observation point and is situated in the near area of the dipole. As opposite to [1] and [2] we do not use any simplifications concerning the problem geometry.

The magnitude of position vectors are as follows

$$
\begin{aligned}
r & =\sqrt{x^{2}+y^{2}+z^{2}} \\
R & =\sqrt{x^{2}+y^{2}+\left(z^{\prime}-z\right)^{2}} \\
R_{1} & =\sqrt{x^{2}+y^{2}+(h-z)^{2}} \\
R_{2} & =\sqrt{x^{2}+y^{2}+(h+z)^{2}}
\end{aligned}
$$

Now, using complex representation, let us suppose the sinusoidal current distribution as in [2]

$$
\begin{aligned}
\mathcal{J}_{z}\left(z^{\prime}\right)=\mathcal{I}_{0} \sin \left(k\left(h-z^{\prime}\right)\right) & & z>0 \\
\mathcal{J}_{z}\left(z^{\prime}\right)=\mathcal{I}_{0} \sin \left(k\left(h+z^{\prime}\right)\right) & & z<0
\end{aligned}
$$
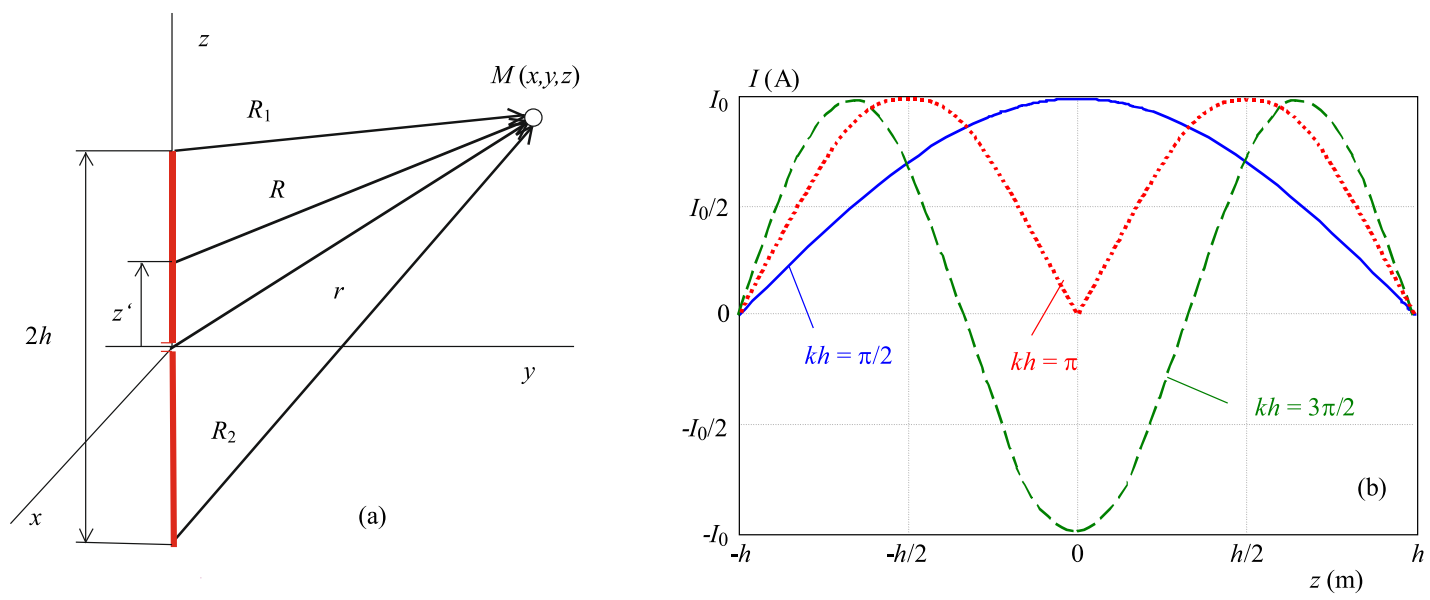

Fig. 1. (a) - dipole geometry for near-field analysis according to (1), and (b) - the current distribution according to (2)

* Slovak University of Technology, Faculty of Electrical Engineering and Information Technology, Institute of Electrical Engineering, Ilkovičova 3, 81219 Bratislava 1, Slovakia, rene.hartansky@stuba.sk 
where $\mathcal{J}_{0}$ is the maximal current phasor along the the dipole antenna (the air gap being neglected) and $k$ - is the wave number a real value in a lossless media.

The complex vector potential $\mathcal{A}$ pertaining to the above given current distribution, from which the field components can be expressed, is

$$
\begin{aligned}
\mathcal{A}_{z}= & \frac{\mu \mathcal{J}_{0}}{4 \pi}\left\{\int_{0}^{h} \sin \left(k\left(h-z^{\prime}\right)\right) \frac{e^{-j k R}}{R} \mathrm{~d} z^{\prime}+\right. \\
& \left.\int_{-h}^{0} \sin \left(k\left(h+z^{\prime}\right)\right) \frac{e^{-j k R}}{R} \mathrm{~d} z^{\prime}\right\}
\end{aligned}
$$

what, using Euler's formulae, can be written as

$$
\mathcal{A}_{z}(z)=\frac{\mu_{0} \mathcal{J}_{0}}{j 8 \pi} \sum_{\nu=1}^{4} \mathcal{I}_{\nu}
$$

where

$$
\begin{aligned}
& \mathcal{I}_{1}=e^{j k h} \int_{0}^{h} \frac{e^{-j k\left(\sqrt{x^{2}+y^{2}+\left(z-z^{\prime}\right)^{2}}+z^{\prime}\right)}}{\sqrt{x^{2}+y^{2}+\left(z-z^{\prime}\right)^{2}}} \mathrm{~d} z^{\prime} \\
& \mathcal{I}_{2}=e^{-j k h} \int_{0}^{h} \frac{e^{-j k\left(\sqrt{x^{2}+y^{2}+\left(z-z^{\prime}\right)^{2}}-z^{\prime}\right)}}{\sqrt{x^{2}+y^{2}+\left(z-z^{\prime}\right)^{2}}} \mathrm{~d} z^{\prime} \\
& \mathcal{I}_{3}=e^{j k h} \int_{-h}^{0} \frac{e^{-j k\left(\sqrt{x^{2}+y^{2}+\left(z-z^{\prime}\right)^{2}}-z^{\prime}\right)}}{\sqrt{x^{2}+y^{2}+\left(z-z^{\prime}\right)^{2}}} \mathrm{~d} z^{\prime} \\
& \mathcal{I}_{4}=e^{-j k h} \int_{-h}^{0} \frac{e^{-j k\left(\sqrt{x^{2}+y^{2}+\left(z-z^{\prime}\right)^{2}}+z^{\prime}\right)}}{\sqrt{x^{2}+y^{2}+\left(z-z^{\prime}\right)^{2}}} \mathrm{~d} z^{\prime}
\end{aligned}
$$

Since the magnetic field is determined by rotation of vector potential: $\overrightarrow{\mathcal{H}}=\operatorname{rot} \overrightarrow{\mathcal{A}} / \mu$, in Cartesian coordinate system, due to existence of $\mathcal{A}_{z}$ component only, we have

$$
\mathcal{H}_{x}=\frac{1}{\mu} \frac{\partial \mathcal{A}_{z}}{\partial y}, \mathcal{H}_{y}=-\frac{1}{\mu} \frac{\partial \mathcal{A}_{z}}{\partial x} .
$$

Further we confine ourselves only to $x$-axes components of the magnetic field ( $y$-axes component will be calculated similarly) using (4) and (5). Now, if the rotation will precede the integration we get

$$
\begin{aligned}
\frac{\partial \mathcal{I}_{1}}{\partial y} & =e^{j k h} \int_{0}^{h} \frac{\partial}{\partial y} \frac{e^{-j k\left(R+z^{\prime}\right)}}{R} \mathrm{~d} z^{\prime} \\
& =e^{j k h} \int_{0}^{h} y(-1-j k R) \frac{e^{-j k\left(R+z^{\prime}\right)}}{R^{3}} \mathrm{~d} z^{\prime} \\
\frac{\partial \mathcal{I}_{2}}{\partial y} & =e^{-j k h} \int_{0}^{h} y(-1+j k R) \frac{e^{-j k\left(R-z^{\prime}\right)}}{R^{3}} \mathrm{~d} z^{\prime} \\
\frac{\partial \mathcal{I}_{3}}{\partial y} & =e^{j k h} \int_{0}^{h} y(-1-j k R) \frac{e^{-j k\left(R+z^{\prime}\right)}}{R^{3}} \mathrm{~d} z^{\prime} \\
\frac{\partial \mathcal{I}_{4}}{\partial y} & =e^{-j k h} \int_{0}^{h} y(-1+j k R) \frac{e^{-j k\left(R-z^{\prime}\right)}}{R^{3}} \mathrm{~d} z^{\prime}
\end{aligned}
$$

According to [1], the primitive functions of the above integrals are known and so the first and the third and the second and fourth expressions in (7) give

$$
\begin{aligned}
& \mathcal{P}_{1}\left(z^{\prime}\right)=\frac{y e^{-j k\left(R+z^{\prime}\right)}}{R\left(R+z^{\prime}-z\right)}, \\
& \mathcal{P}_{2}\left(z^{\prime}\right)=\frac{y e^{-j k\left(R-z^{\prime}\right)}}{R\left(R-z^{\prime}+z\right)}
\end{aligned}
$$

and to express $x$ - component of the magnetic field using (4) and (6) after some manipulation we can put down

$$
\begin{aligned}
\mathcal{H}_{x}=\frac{\mathcal{J}_{0}}{j 8 \pi} & \left\{e^{j k h}\left[\mathcal{P}_{1}(h)-\mathcal{P}_{1}(0)\right]\right. \\
& +e^{-j k h}\left[\mathcal{P}_{2}(h)-\mathcal{P}_{2}(0)\right] \\
& +e^{j k h}\left[\mathcal{P}_{2}(-h)-\mathcal{P}_{2}(0)\right] \\
& \left.+e^{-j k h}\left[\mathcal{P}_{1}(-h)-\mathcal{P}_{1}(0)\right]\right\}
\end{aligned}
$$

after putting (8) into (9) and relaying on forms (1) and processing in the same way also the other component we arrive to

$$
\begin{gathered}
\mathcal{H}_{x}=-j \frac{\mathcal{J}_{0}}{4 \pi} \frac{y}{x^{2}+y^{2}}\left\{e^{-j R_{1}}+e^{-j k R_{2}}\right. \\
\left.-e^{-j k r}\left[e^{-j k h}\left(1+e^{2 j k h}\right)\right]\right\}= \\
=-j \frac{\mathcal{J}_{0}}{4 \pi} \frac{y}{x^{2}+y^{2}}\left\{e^{-j R_{1}}+e^{-j k R_{2}}\right. \\
\left.\quad-2 e^{-j k r} \cosh (k h)\right\} \\
\mathcal{H}_{y}=j \frac{\mathcal{J}_{0}}{4 \pi} \frac{y}{x^{2}+y^{2}}\left\{e^{-j R_{1}}+e^{-j k R_{2}}\right. \\
\left.\quad-2 e^{-j k r} \cosh (k h)\right\} .
\end{gathered}
$$

The electric field of the electrical dipole can be determined directly from the Maxwell equation, using $\overrightarrow{\mathcal{B}}=$ $\operatorname{rot} \overrightarrow{\mathcal{A}}$, from

$$
\overrightarrow{\mathcal{E}}=\frac{1}{j \omega \epsilon} \operatorname{rot} \overrightarrow{\mathcal{A}}
$$

giving the three components of the electric field in Cartesian coordinate system

$$
\begin{array}{r}
\mathcal{E}_{x}=\frac{1}{j \omega \epsilon}\left(\frac{\partial \mathcal{H}_{z}}{\partial y}-\frac{\partial \mathcal{H}_{y}}{\partial z}\right)= \\
\frac{j \mathcal{J}_{0} x k}{4 \pi \omega \epsilon\left(x^{2}+y^{2}\right)}\left\{(h-z) \frac{e^{-j k R_{1}}}{R_{1}}\right. \\
\left.-(h+z) \frac{e^{-j k R_{2}}}{R_{2}}+2 z \frac{e^{-j k r}}{r} \cos (k h)\right\} \\
\mathcal{E}_{y}=\frac{1}{j \omega \epsilon}\left(\frac{\partial \mathcal{H}_{x}}{\partial z}-\frac{\partial \mathcal{H}_{z}}{\partial x}\right)=
\end{array}
$$



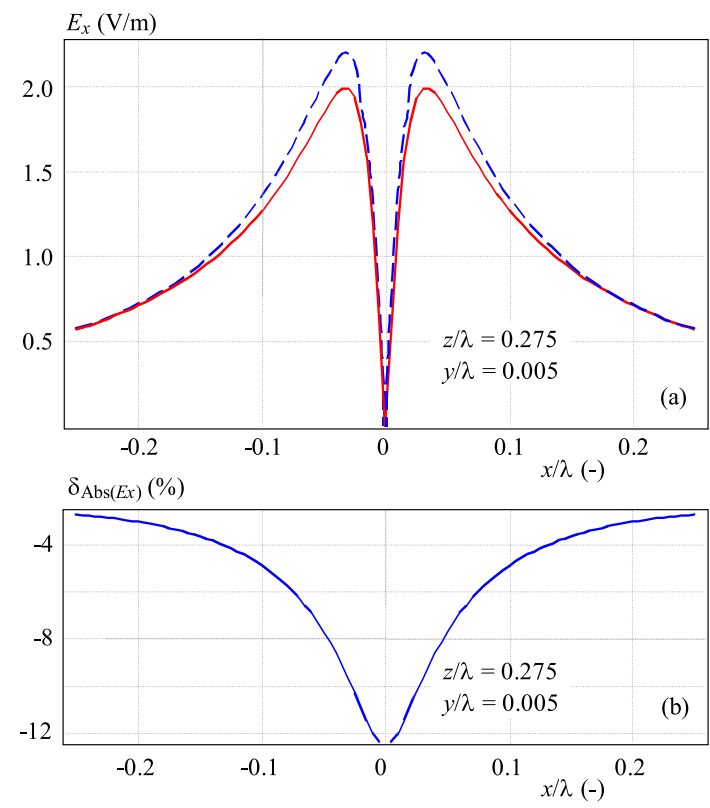

Fig. 2. (a) - amplitude of $x$-axes component of the electric field as a function of $x$ and, (b) - the error related to numerical computation FEKO
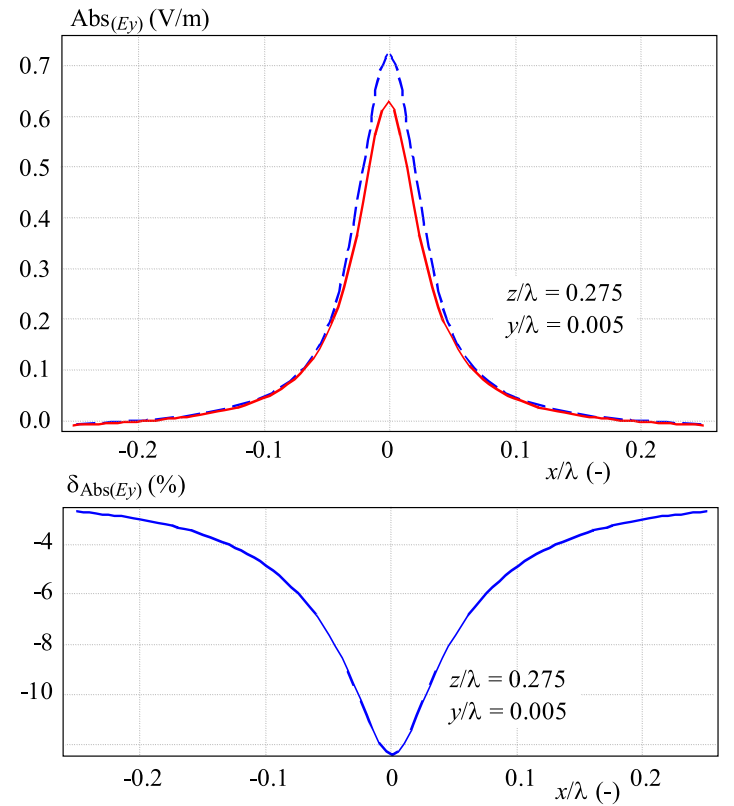

Fig. 4. (a) - amplitude $y$-axes component of the electric field as a function of $x$, and (b) - the error related to numerical computation FEKO

$$
\begin{array}{r}
\frac{j \mathcal{J}_{0} y k}{4 \pi \omega \epsilon\left(x^{2}+y^{2}\right)}\left\{(h-z) \frac{e^{-j k R_{1}}}{R_{1}}\right. \\
\left.-(h+z) \frac{e^{-j k R_{2}}}{R_{2}}+2 z \frac{e^{-j k r}}{r} \cos (k h)\right\} \\
\mathcal{E}_{z}=\frac{1}{j \omega \epsilon}\left(\frac{\partial \mathcal{H}_{y}}{\partial x}-\frac{\partial \mathcal{H}_{x}}{\partial y}\right)=
\end{array}
$$
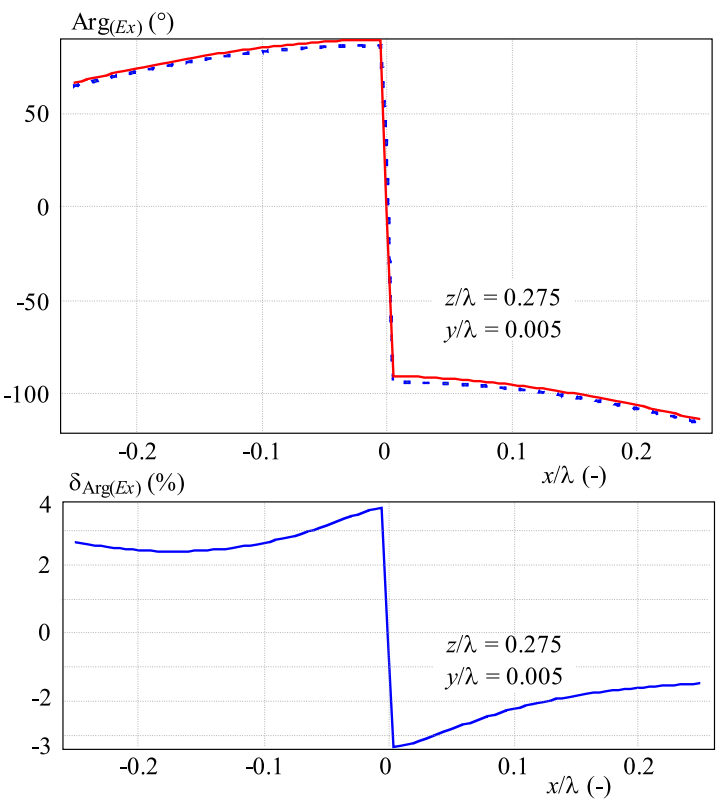

Fig. 3. (a) - phase of $x$-axes component of the electric field as a function of $x$, and (b) - the error related to numerical computation FEKO
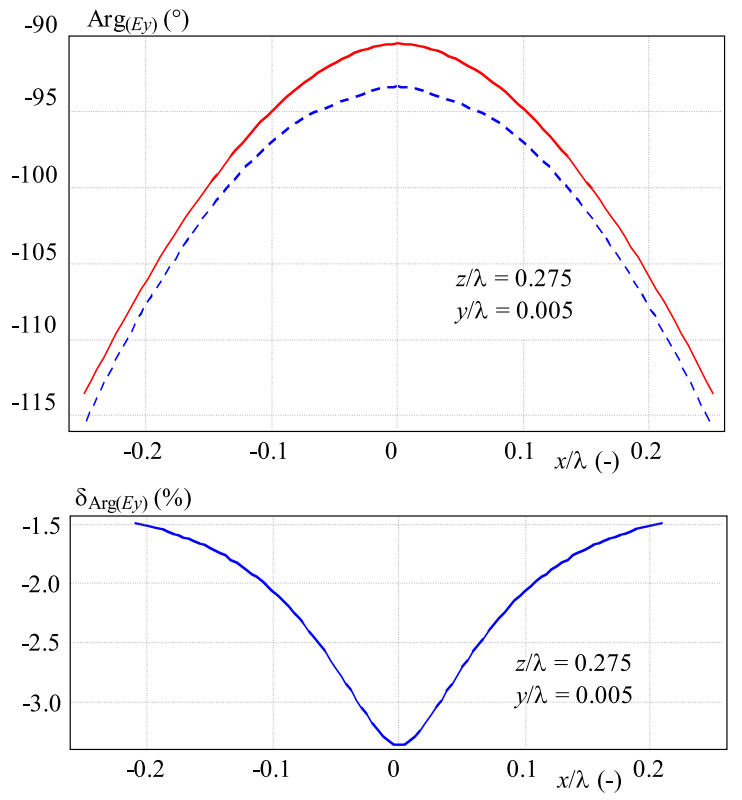

Fig. 5. (a) - phase of $y$-axes component of the electric field as a function of $x$, and (b) - the error related to numerical computation FEKO

$$
\begin{array}{r}
\frac{j \mathcal{J}_{0} z k}{4 \pi \omega \epsilon\left(x^{2}+y^{2}\right)}\left\{(h-z) \frac{e^{-j k R_{1}}}{R_{1}}\right. \\
\left.-(h+z) \frac{e^{-j k R_{2}}}{R_{2}}+2 z \frac{e^{-j k r}}{r} \cos (k h)\right\} .
\end{array}
$$

In the above equations it holds

$$
\frac{k}{4 \pi \omega \epsilon}=\frac{\sqrt{\mu / \epsilon}}{4 \pi}
$$

giving in vacuum value of 30 . 


\section{VERIFICATION}

The final equation of component of the electric field $\mathcal{E}_{z}$ is obviously the same in Cartesian coordinate system as in the cylindrical coordinate system. The electric component of radiated field $\mathcal{E}_{z}$ can be found elswhere, for instance also in [1] and [2], however in the Cartesian coordinate system the closed form of electric components $\mathcal{E}_{x}$ and $\mathcal{E}_{y}$ are hard to find in available literature. To verify the correctness of $\mathcal{E}_{x}$ and $\mathcal{E}_{y}$ calculation a numerical Method of moments was used as implemented in commercial software FEKO. For these calculations of the field components of a conductive element, the half-wave dipole configuration with negligible radius was used.

In Fig. 2 a comparison of the numerically obtained $x$ axes component of the near electric field (ringed line) and closed analytical calculation of its magnitude is shown. We see that the waveforms are almost identical relating the shape and the values as documented in Fig. 2(b). Because that software FEKO allows only voltage feeding of dipole it was necessary to modify current in equation (12) to get identical initial conditions for numerical and analytical calculation.

A plot of the phase of $x$-axes near electric field component shown in Fig. 3, possesses the close similarity as well, the shape of analytical and numerical calculations is almost the same.

Next the verification of analytical and numerical solutions are presented for the $y$-axis component of electric field as a dependence on $x$ - coordinate, of namplitude Fig. 4, and phase Fig. 5.

\section{DISCUSSION}

In this article have been analytical expressed radiated components of the electromagnetic field of the conductive element half-wave dipole. The solution of the radiated electromagnetic field is known for cylindrical coordinate system and describe in literature. The electromagnetic near field expression for the Cartesian coordinate system is not known and not available in literature. Analytical calculation other characteristics (mutual impedance, input impedance, etc.) of two orthogonal conductive elements in the cylindrical coordinate system are difficult or error loaded, because have to used Cartesian coordinate. Analytical solution of electric components of the electromagnetic field was verified by numerical method.

\section{Acknowledgements}

The support of this work by the scientific agency VEGA under projects 1/0963/12 and 2/0048/13 is kindly acknowledged and special thanks to Mojmir Kollar for expert advice.

\section{REFERENCES}

[1] BALANIS, C. A : Antenna theory and design (Third edition), New Jersey: John Wiley \& Sons, 2005, ISBN 0-471-66782-X.

[2] VÁVRA, S.-TURÁN, J.: Antennas and Electromagnetic Wave Propagation (Antény a šírenie elektromagnetickch vín), Bratislava: Alfa, ISBN 80-05-00131-2, in Slovak.

[3] SLÍŽIK, J.-HARŤANSKÝ, R. : Quality Innovation and Prosperity 17(1), 57-66, ISSN 1335-1745.

Received 3 November 2012

René Hart'anský (doc, Ing, PhD) was born in Bansk Bystrica, Slovak Republic, in 1969. He received the Ing degree in 1992 and the $\mathrm{PhD}$ degree in measurement technique from the Faculty of Electrical Engineering of the Slovak Technical University (FEE STU) in 1999. He is currently the associate professor at the Institute of electrical engineering of the STU Bratislava. His research interests are the computer modeling EMC phenomena, antennas and propagation.

Jozef Slížik (Ing), is a PhD student, Faculty of Electrical Engineering and Information Technology, Slovak University of Technology in Bratislava, Slovakia.

Lukáš Maršálka (Ing), was born in Trenn, Slovakia, in 1986. He graduated from the Faculty of Mechatronics, Alexander Dubček University of Trenčí. At present he is a PhD student at the Institute of Electrical Engineering, Faculty of Electrical Engineering and Information Technology. The main field of his research is con-tactless distance measurement and measurement of electrical quantities. 